\title{
ULTRAVIOLET POLARIZATION MEASUREMENTS OF MARS AND THE OPACITY OF THE MARTIAN ATMOSPHERE*
}

\author{
ANDREW P. INGERSOLL \\ Division of Geological Sciences, California Institute of Technology, Pasadena, Calif., U.S.A.
}

\begin{abstract}
Ground-based polarimetric data taken near maximum elongation are presented. These data are analyzed assuming an optically thin, Rayleigh scattering atmosphere, and a surface whose polarization varies inversely as the surface albedo. The best fit to the data yields an optical depth for Rayleigh scattering corresponding to a surface pressure of $6 \pm 1 \mathrm{mb}$, if carbon dioxide is the principal constituent. There is no need to postulate the existence of fine dust in the Martian atmosphere. This method is potentially capable of resolving elevation differences on the Martian surface.
\end{abstract}

\section{Introduction}

This paper is a report of polarization measurements of Mars at $200 \AA$ resolution from $3200 \AA$ to $7000 \AA$ wavelength. The measurements were made near maximum elongation, when the solar phase angle $\theta$ was $42.5^{\circ}$. Two quantities were determined for each wavelength interval: the percentage polarization of reflected sunlight from a region of Mars, and the direction of polarization relative to the Sun-Mars line in the terrestrial sky. Combining these data with the photometric brightness measurements of Irvine et al. (1968) yields an estimate of the optical depth for Rayleigh scattering on Mars. The analysis is based on the assumption of an optically thin, Rayleigh scattering atmosphere, and a surface of low albedo which polarizes inversely as its albedo. The derived value of surface pressure is in accord with the Mariner occultation estimates, and the probable error is no larger than that obtained from the occultations. Thus there is no inconsistency between these polarization measurements and the model of a pure Rayleigh scattering atmosphere of carbon dioxide.

The significance of this conclusion is first, that previous polarization studies have been cited as evidence for high surface pressures (Pollack, 1967), or fine dust in the Martian atmosphere (Dollfus and Focas, 1966), but in fact, the polarization measurements are consistent with a simpler model in agreement with spacecraft results. The main difference between these measurements and previous measurements is the full wavelength coverage from 3200 to $7000 \AA$. Data at the shorter wavelengths are especially important because the atmosphere is the dominant source of polarization only at wavelengths below $4000 \AA$. The second aspect of these results is that the method may be used to measure topographic relief on Mars, as an alternative to the radar method (Pettengill et al., 1969) and the spectroscopic method (Belton and Hunten, 1969). The method may also be used in searching for an atmosphere on Mercury.

* Contribution No. 1694 of the Division of Geological Sciences, California Institute of Technology. 


\section{Experimental Procedure}

The measurements were made on August 12-13, 1969 from 03 30-0530 UT, with the 100-inch telescope on Mt. Wilson. The instrument was operated at the Cassegrain focus, off the axis of the telescope, in such a way that the polarization introduced by the reflection at the Cassegrain flat was cancelled by reflection at a second mirror in the photometer (Figure 1). Thus, letting the axis of the telescope be the $x$-axis and the deflected beam be the $y$-axis, the direction after the second reflection must be the $z$-axis in order that the polarizations cancel. It is also necessary that both mirrors be of the same material (i.e., aluminum).

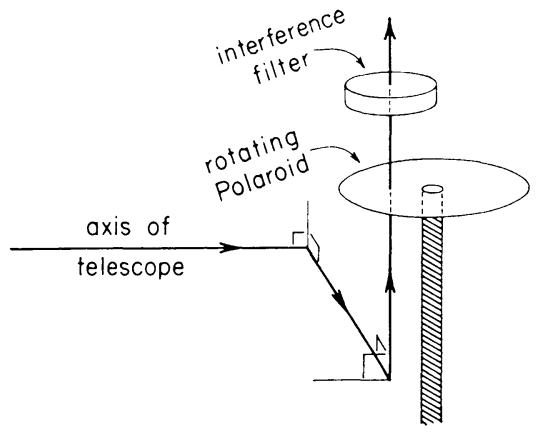

Fig. 1. Ray path in photoelectric polarimeter for use at East- or West-arm Cassegrain focus.

After the second right-angle reflection, the beam passed through a rotating Polaroid disk, then through an interference filter, and then into the detector, an S-20 photomultiplier tube. The a.c. component of the signal was synchronously demodulated using a phase reference driven at twice the frequency of the Polaroid disk. (A $180^{\circ}$ rotation of the Polaroid corresponds to a full cycle in the plane of polarization.) The d.c. component of the signal was amplified separately, and both outputs were simultaneously recorded on a d.c. strip chart recorder.

If we think of the incoming flux as the superposition of two linearly polarized fluxes $F^{\perp}$ and $F^{\|}$, then the output of the d.c. channel is proportional to $\left(F^{\perp}+F^{\|}\right)$, and the a.c. channel to $\left(F^{\perp}-F^{\|}\right) \times \cos \psi$, where $\psi$ is the phase of the reference signal relative to the $\perp$ position of the Polaroid. This is equivalent to resolving the polarized flux $\left(F^{\perp}-F^{\prime \prime}\right)$ along a direction at an angle $\psi / 2$ to the $\perp$ direction. Thus measurements at two values of $\psi$ are sufficient to determine the plane of polarization and degree of polarization of the incident beam. The system was calibrated in advance using planepolarized light in known orientations.

This system was tested using stars whose polarization has been measured or which have degrees of polarization below some threshold value (Hall, 1958; Appenzeller, 1968). The probable error determined in this way was several tenths of $1 \%$ in the degree 
of polarization, and several degrees of angle in the plane of polarization. Since the measured polarizations varied from $1.3 \%$ at $7000 \AA$, to $13 \%$ at $3200 \AA$, this was considered sufficient accuracy for the purposes of the experiment.

\section{Analysis of Results}

Measurements were made of a large bright area (Arabia) at Martian longitude $290^{\circ}$ to $330^{\circ}$, which was located in the center of the disk at the time of observation. The solar phase angle was $42.5^{\circ}$. The plane of polarization was found to lie perpendicular to the Sun-Mars line at wavelengths less than $4500 \AA$, but appeared to deviate up to $10^{\circ}$ at longer wavelengths. The observed degree of polarization vs wavelength is plotted in Figure 2.

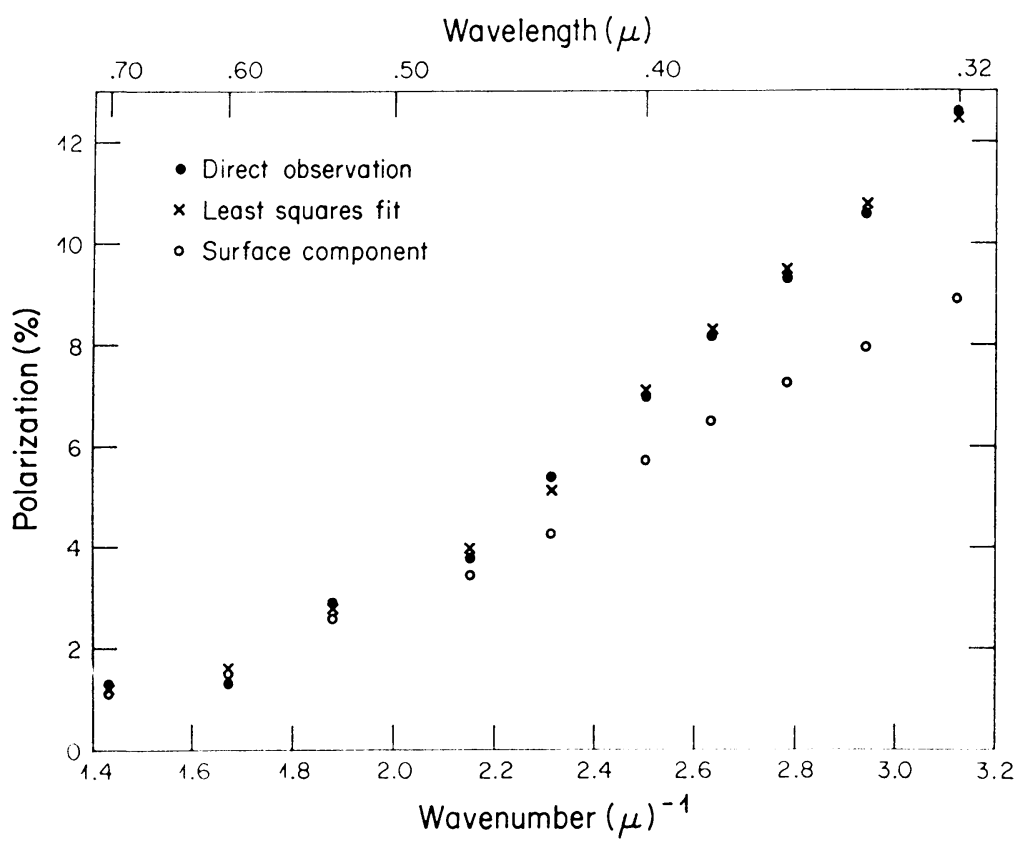

Fig. 2. Polarization of Arabia at the center of the Martian disk for solar phase angle $42.5^{\circ}$.

The most important part of the data analysis is separating effects of the surface from those of the atmosphere. To do this, we need a model which predicts how each component of the reflected light should vary with wavelength, and how these components should combine to produce the observed signal. The simplest model of the atmosphere is a layer of Rayleigh scatterers, i.e., particles small compared to the wavelength. This model includes the case of a pure, molecular atmosphere, or one with very fine suspended particles. We shall also assume that the atmosphere is optically thin, which follows from the Rayleigh scattering assumption and the fact that the 
planet has a low albedo. The attenuation of the incident solar beam as it passes through the atmosphere will be neglected; only first-order scattering, directly out of the incident beam, will be considered. Illumination of the atmosphere by the surface, and vice-versa, will be neglected because the albedo of the surface and the optical depth of the atmosphere are both small.

The polarization measurements described here refer to a point on the disk defined by $\mu_{0}$ and $\mu$, the cosines of the angles of incidence and reflection, respectively. In analysing the data, it is necessary to know the absolute value of the intensity from the point, as well as its direction and degree of polarization. Unfortunately, however, most of the absolute brightness studies refer to the integrated disk, at various wavelengths and phase angles. Therefore, we need a scattering law for the surface and atmosphere in order to obtain the brightness of a point of the disk from the average brightness of the whole planet.

For an optically thin, Rayleigh scattering atmosphere, the scattering law is known. We define the optical depth

$$
\tau=\frac{8 \pi^{3}}{3 \lambda^{4}} \frac{\left(n^{2}-1\right)^{2}}{N_{0}^{2}} \int_{0}^{\infty} N \mathrm{~d} z,
$$

where $\lambda$ is the wavelength of the scattered light, $n$ is the index of refraction of the medium at STP, $N_{0}$ is Loschmidt's number, $z$ is the vertical coordinate, and $N$ is the number density of molecules in the atmosphere. For a point $\left(\mu_{0}, \mu\right)$ the intensity of light scattered by the atmosphere is

$$
I_{a}^{\perp}=\frac{3 F_{\odot} \tau}{16 \pi \mu}, \quad I_{a}^{\|}=\frac{3 F_{\odot} \tau}{16 \pi \mu} \cos ^{2} \theta
$$

in a specified wavelength interval, where $F_{\odot}$ is the flux of sunlight at Mars in the interval. The symbols $\perp$ and $\|$ refer to components resolved along directions perpendicular and parallel to the plane of scattering, and the subscripts ' $a$ ' refer to the atmosphere. Equation (2) is singular at the limb, but the singularity is integrable, and the equation may be integrated over the illuminated portion of the disk to yield the flux per unit solid angle emanating from the atmosphere:

$$
F_{a}^{\perp}+F_{a}^{\sharp}=\frac{3 F_{\odot} \tau}{8 \pi}(\pi-\theta)\left(1+\cos ^{2} \theta\right) r^{2},
$$

where $r$ is the radius of the planet. Finding $\tau$ is our main task, but first we must develop a set of relations for the surface, similar to Equations (2)-(3).

We assume a surface scattering law of the form (Minnaert, 1941; Harris, 1961)

$$
I_{s}^{\perp}+I_{s}^{\mathbb{Q}}=F_{\odot} C \mu_{0}^{k} \mu^{k-1} .
$$

Here $k$ will be regarded as a parameter of the problem independent of wavelength; the analysis is insensitive to the value of $k$ for $0.75 \leqslant k \leqslant 1.0$. The quantity $C$ is related to the brightness of the surface, and is therefore wavelength dependent. Integrating (4) 
over the illuminated disk we obtain, for the flux per unit solid angle emanating from the surface:

$$
F_{s}^{\perp}+F_{s}^{\|}=F_{\odot} r^{2} C \int_{\theta-(\pi / 2)}^{\pi / 2}[\cos (\phi-\theta) \cos \phi]^{k} \mathrm{~d} \phi \int_{-1}^{+1}\left(1-\mu^{2}\right)^{k} \mathrm{~d} \mu .
$$

Then, combining Equations (3) and (5) and dividing by $r^{2} F_{\odot}$, we obtain an expression for the geometric albedo of the planet at phase $\theta$ in terms of $\tau, k$, and $C$. The geometric albedo of Mars $p(\lambda, \theta)$ has been measured at the wavelengths and phases of interest in this study (Irvine et al., 1968) and therefore we may express $C$ in terms of $\tau$ and $k$, for each wavelength of interest:

$$
C=\frac{p(\lambda, \theta)-(3 \tau / 8 \pi)(\pi-\theta)\left(1+\cos ^{2} \theta\right)}{\int_{\theta-(\pi / 2)}^{\pi / 2}[\cos (\phi-\theta) \cos \phi]^{k} \mathrm{~d} \phi \int_{-1}^{1}\left(1-\mu^{2}\right)^{k} \mathrm{~d} \mu} .
$$

Finally, an important assumption of the analysis is that the degree of polarization of light scattered by the surface is inversely proportional to $C$, that is, inversely proportional to surface brightness. This law appears to hold for the Moon (Gehrels et al., 1964), and for powders which have been observed in the laboratory (Dollfus, 1961 ; Coffeen, 1965). Thus we assume

$$
I_{s}^{\perp}-I_{s}^{\|}=F_{\odot} \beta \mu_{0}^{k} \mu^{k-1},
$$

where $\beta$ is a constant, like $k$, which is independent of wavelength. The degree of polarization from the surface $\delta_{s}$ is $\beta / C$, which follows from Equations (4) and (7).

The polarized component of intensity from a point on the disk is obtained by combining $I_{a}^{\perp}-I_{a}^{\|}$and $I_{s}^{\perp}-I_{s}^{\|}$, Equations (2) and (7), respectively. Dividing this by the total intensity we obtain an expression for the degree of polarization of a point on the disk:

$$
\delta=\frac{(3 \tau / 16 \pi \mu)\left(1-\cos ^{2} \theta\right)+\beta \mu_{0}^{k} \mu^{k-1}}{(3 \tau / 16 \pi \mu)\left(1+\cos ^{2} \theta\right)+C \mu_{0}^{k} \mu^{k-1}}
$$

Equation (8) involves the undetermined quantities $\tau, \beta, k$, and $C$. However $C$ can be eliminated from the problem by Equation (6), and $k$ is treated as a parameter in the range $0.75 \leqslant k \leqslant 1.0 . \tau$ varies as $\lambda^{-4}$ and $\beta$ is a constant independent of wavelength, so the problem reduces to determining the two constants $\tau \lambda^{4}$ and $\beta$.

The experimental data in Figure 2 refer to a large bright area centered at $\mu_{0}=0.91$, $\mu=0.95$. Using these values, the degree of polarization $\delta$ was computed from Equation (8) for various $\tau \lambda^{4}$ and $\beta$. For each $\tau$, values of $C$ were determined from Equation (6), using determinations of $p(\lambda, \theta)$ from Irvine et al. (1968). It is necessary to assume that the area where the polarization measurements were made is typical of the planet as a whole; this is probably valid for bright areas, which comprise about $70 \%$ of the area of Mars. In this way, the values of $\tau \lambda^{4}$ and $\beta$ which produce the best least-squares fit to the observational data in Figure 2 are

$$
\beta=0.9 \times 10^{-3}, \quad \tau \lambda^{4}=2.1 \times 10^{-4}(\mu \mathrm{m})^{4} .
$$


The uncertainty in each of the above quantities is about $\pm 15 \%$, which includes the probable error from the least-squares fit for a given $k$, as well as the variation for different $k$ in the range $0.75 \leqslant k \leqslant 1.0$. The points calculated in this way are also plotted in Figure 2.

\section{Conclusions}

We may interpret the estimate of $\tau \lambda^{4}$ in Equation (9) assuming the Rayleigh scattering opacity is due entirely to an atmosphere of pure carbon dioxide. This yields an estimate of the number of molecules in an atmospheric column, and therefore of the Martian surface pressure $P_{s}$ on the region of Mars where the measurements were made:

$$
P_{s}=6 \pm 1 \mathrm{mb} \text {. }
$$

From the derived values of $\beta$ and $C$ we may compute the polarization of light scattered by the surface alone, $\delta_{s}=\beta / C$. These values are also plotted in Figure 2 .

As stated in the Introduction, the interpretation presented here is based on the simplest model one might propose for the Martian atmosphere. Other models could be made to fit the data equally well, but the agreement between our estimate of surface pressure Equation (10), and the generally accepted value, is significant. Our conclusion is that polarization measurements are consistent with a molecular atmosphere without suspended fine particles. On the other hand, thin clouds are observed in the Martian atmosphere (Leighton et al., 1969), but these do not necessarily contribute to the observed polarization. If the light scattered by these clouds is unpolarized, their presence will have no effect on the present interpretation.

The optical depth reported here is small even at the shortest wavelengths, and therefore the atmosphere is quite transparent. If this model is correct, then the disappearance of surface features in the blue is a property of the surface itself, and is not due to obscuration by the atmosphere. On the other hand, the light scattered by the atmosphere is a significant fraction of the total light from the planet at the shortest wavelengths, because the surface albedo is low. According to our model, the surface albedo decreases to $60 \%$ of the planetary albedo at $3200 \AA$, and so the surface must be extremely dark in the ultraviolet.

The results reported in this paper are preliminary. Future observations should include direct measurements of brightness and polarization across the disk, and should be analysed to include effects associated with observed surface features, clouds, and pressure variations associated with surface topography. The present study indicates the usefulness of polarization measurements in the ultraviolet, in investigations of thin planetary atmospheres.

\section{Acknowledgments}

I should like to thank James A. Westphal for much advice, encouragement and instruction in various aspects of observational astronomy, and Sol. L. Giles for help in building the equipment used in this study.

13-P.A. 


\section{References}

Appenzeller, I.: 1968, Astrophys. J. 151, 907.

Belton, M. J. S. and Hunten, D. M.: 1969, Science 166, 225.

Coffeen, D. L.: 1965, Astron. J. 70, 403.

Dollfus, A.: 1961, in Planets and Satellites (ed. by G. P. Kuiper and B. M. Middlehurst), University of Chicago Press, Chicago, p. 343.

Dollfus, A. and Focas, J.: 1966, Compt. Rend. Acad. Sci. Paris B262, 1024.

Gehrels, T. et al.: 1964, Astron. J. 69, 826.

Hall, J. S.: 1958, Publ. U.S. Naval Observatory, 2nd Ser. 17, 271.

Harris, D. L.: 1961, in Planets and Satellites (ed. by G. P. Kuiper and B. M. Middlehurst), University of Chicago Press, Chicago, p. 272.

Irvine, W. M. et al.: 1968, Astron. J. 73, 807.

Leighton, R. B. et al.: 1969, Science 166, 49.

Minnaert, M.: 1941, Astrophys. J. 93, 403.

Pettengill, G. H. et al.: 1969, Astron. J. 74, 461.

Pollack, J. B.: 1967, Icarus 7, 42. 